\title{
Câncer Ano-Reto-Cólico: Aspectos Atuais IV - Câncer de Cólon - Fatores Clínicos, Epidemiológicos e Preventivos
}

\author{
Anal Canal and Colorectal Cancer: Current Features: IV - Colon Cancer - \\ Clinical, Epidemiological, and Preventive Aspects
}

\author{
JÚLIO CÉSAR M SANTOS JR. ${ }^{1}$ \\ ${ }^{1}$ Membro Titular da Sociedade Brasileira de Coloproctologia.
}

\begin{abstract}
SANTOS JR JCM. Câncer Ano-Reto-Cólico: Aspectos Atuais IV - Câncer de Cólon - Fatores Clínicos, Epidemiológicos e Preventivos. Rev bras Coloproct, 2008;28(3): 378-385.

RESUMO: O câncer colorretal tem alta incidência populacional e alto índice de mortalidade, com diferenças pouco relevantes entre os povos de diversas nações, como atestam os estudos epidemiológicos dessa doença. Embora a abordagem médico-cirúrgica do câncer colorretal tenha sido favorecida pelos novos conhecimentos adquiridos com a engenharia genética, pelos progressos que aprimoraram o tratamento, principalmente na área de neo-adjuvância, com as inovações nos aparelhos de radioterapia e com a constante introdução de novas e potentes substâncias quimioterápicas, o prognóstico da doença continua sombrio. Todavia, dados colhidos em estudos sobre a biologia do tumor - sua origem, crescimento e desenvolvimento e comportamento biológico - têm acenado para a possibilidade de cura quando os métodos preventivos, em prática, facilitam a abordagem precoce da lesão. Nesse contexto, o câncer colorretal é passível de cura, podendo, inclusive dispensar, para tanto, o tratamento adjuvante ou aliviar o paciente da abordagem cirúrgica mutilante. Assim, o maior esforço posto em ação no início desse século está sendo representado pelos movimentos de educação popular em massa para a prevenção do câncer de reto e dos cólons com incentivo para o teste de sangue oculto nas fezes.
\end{abstract}

Descritores: câncer, câncer colorretal, câncer de cólon, epidemiologia, medidas preventivas, sangue oculto, fezes.

\section{INTRODUÇÃO-ASPECTOS CLÍNICOS E EPIDEMIOLÓGICOS}

A ocorrência estimada de câncer de cólon, para 2008, nos Estados Unidos foi de 108.070 casos novos que somados aos previstos 40.740 cânceres de reto para o mesmo ano, num total de 148.810 casos, classifica o adenocarcinoma colorretal em terceiro lugar dentre os mais comuns, afetando tanto os homens como as mulheres ${ }^{1,2}$, tal como na Europa ${ }^{3}$.

No Brasil, embora publicada pelo Inca, a estatística não é de fácil acesso; tem discrepâncias notáveis que parecem expressar distorções regionais o que tornam pouco aceitáveis os números relatados ${ }^{4,5}$.

Apesar do que se observa nos Estados Unidos, a taxa de incidência tem diminuído nas últimas décadas - avaliada em 66,3/100.000 pessoas, em 1985, caiu para 48,2, em 2004. Esse declínio foi mais acentuado nos últimos anos, provavelmente por causa das campanhas e das buscas ativas, que permitiram as descobertas e retiradas precoces de pólipos antes da transformação maligna ${ }^{1}$.

Dados semelhantes têm sido observados em relação à mortalidade cuja taxa de declínio foi de 1,8\% por ano, no período compreendido entre 1985-2002, para $4,7 \%$ entre 2002 e 2004 , refletindo o melhor tratamento, principalmente em termos de abordagem antecipada, reflexo da mais eficiente prevenção ${ }^{1,6}$, não só fazendo a detecção precoce, nos casos em que o câncer está na fase mais inicial de desenvolvimento, como a detecção prévia em que a lesão atingida é, ainda, um adenoma. Além disso, a enfática descrição e divulga-

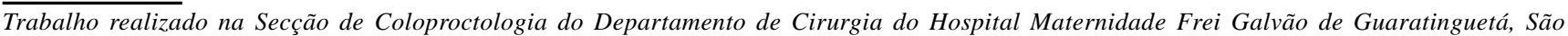
Paulo - Brasil. 
ção dos fatores de risco, mormente os que são passíveis de ser modificados ${ }^{7}$, a elucidação popular por meio de campanhas que divulgam conhecimentos, antes exclusivamente dos médicos ${ }^{8}$, têm formado o conjunto de elementos de proteção e, certamente, contribuído para a diminuição da incidência do câncer colorretal ${ }^{9}$.

Esses e outros aspectos da etiologia do câncer, bem como sobre os elementos facilitadores ou os que agem na dificultação do tratamento já foram discutidos antes ${ }^{5}$.

\section{DIAGNÓSTICO -AVALIAÇÃO E AJUSTES PRÉ-TERAPÊUTICOS}

O mais desfavorável aspecto de implicância com o do câncer de cólon está ligado ao diagnóstico e decorre do silencioso desenvolvimento da lesão e do longo período em que ela permanece assintomática ${ }^{7}$. Outro fator que pode despertar interesse, principalmente porque interfere com elementos da prevenção e do diagnóstico precoce, bem como com o desenvolvimento silencioso do câncer de cólon é a referida tendência da mudança anatômica de distribuição do câncer ao longo intestino grosso. Se o câncer incidir mais no cólon direito, é de se esperar que mais tardia seja a emergência dos primeiro sintomas.

Há mais ou menos 40 anos, o câncer, excetuando o reto, predominava no cólon esquerdo e era mais freqüente no sigmóide, embora as observações a respeito, na época, chamassem a atenção para a ligeira inclinação da curva para a direita. No entanto, quando se comparava a incidência (22\%) de câncer no cólon direito (ceco e ascendente) com a incidência no cólon sigmóide $(45 \%)$ permanecia a idéia da antiga e conhecida distribuição, desse modo, realçando o significante predomínio de um sítio em relação ao outro. Se incluíssemos o cólon descendente e a flexura esplênica o valor percentual da distribuição ascendia para $62 \%$ - considerando todo o intestino grosso, $79 \%$ das lesões estavam no sigmóide e reto e $58 \%$ no reto. Considerando o reto e o cólon sigmóide onde ficam quase $80 \%$ das lesões era possível observar que, numa casuística de 1294 cânceres de reto e de sigmóide, 944 estavam no reto (73\%) e o restante $(27 \%)$ no sigmóide ${ }^{10}$. Entre os localizados no reto, $80 \%$ deles podiam ser tocados. A lesão situada no ceco representava pouco mais que $4 \% .{ }^{10}$

A respeito da distribuição do câncer ao longo dos cólons, os comentários atuais nem sempre são concordantes. Gomez e col. ${ }^{11}$, num estudo feito em
Dewsbury, UK, em 2004, insistiram com a distribuição anatômica do câncer de cólon claramente persistente, sem nenhuma tendência para maior número de tumores no sentido dos segmentos proximais ${ }^{11}$. As distribuições anatômicas evidenciadas seguem o que se observa na disposição dos adenomas ao longo do intestino grosso $^{12}$.

As seguintes informações foram obtidas de estudo da disposição cólica de 2553 pólipos: 1310 (51\%) estavam no cólon esquerdo; 510 (20\%), no cólon direito, mas 733 (29\%) foram síncronos. Entre esses 2533 pólipos, 1659 (65\%) eram adenomas dos quais $44 \%$ estavam no cólon esquerdo e $24 \%$ no cólon direito. Foram diagnosticados 189 carcinomas (7\%), em todos os casos e, nessa circunstância, 71 dos carcinomas $(37,5 \%)$ estavam nos segmentos esquerdos e 118 $(62,5 \%)$ nos segmentos direitos, dado que serviram para apontar o aumento da prevalência de carcinoma em adenomas do lado direito, relacionados com a idade, embora o valor em destaque não tenha sido significativo $(\mathrm{p}=0,0029) .^{12}$

Diante dessas figuras, a despeito de controversas, os autores chamam a atenção para o fato de que as investigações ou rastreamentos de cânceres não podem ser satisfeitos com exames limitados ao cólon esquerdo por causa da perda de $23 \%$ de lesões que estariam localizadas proximais ao ângulo esplênico do cólon ${ }^{12}$, e por causa do notável aumento do câncer nos segmentos proximais ${ }^{13,14}$.

Takada e col. ${ }^{15}$, em 2002, observaram tendência semelhante de aumento do câncer do cólon direito em pacientes japoneses do sexo feminino com idade superior à 70 anos em detrimento da diminuição do câncer de reto, mas isso não se repetiu para população com idade inferior a 69 anos. McFarlane e col. ${ }^{16}$, em estudo feito na Jamaica, detectaram, em 148 pacientes, a seguinte distribuição do câncer de intestino grosso: no cólon direito, 42 pacientes $(28,5 \%)$; no cólon sigmóide, 30 pacientes $(20,3 \%)$; no reto $34(23,2 \%)$ e no cólon esquerdo, exceto o sigmóide, 16 casos $(8,6 \%)$, o que significa uma taxa relativamente baixa para tumores do reto e sigmóide (43,5\%), mostrando a tendência de lateralidade direita para o aparecimento do câncer - diferente de estudos feitos anteriormente na Jamaica - e reforçam a idéia de que os programas de rastreamento do câncer colorretal deve expor todo o intestino grosso.

Na Holanda, entre 1981 e 1996, o câncer do cólon proximal aumentou de 25 para $37 \%{ }^{17}$. 
Quando se comparou brancos e negros americanos, observou-se, entre os negros, que a prevalência de câncer para os segmentos cólicos proximais foi maior que na população branca o que orientou um planejamento diferente quando se propunha programas de rastreamento para aquela e para outras populações ${ }^{18-}$ ${ }^{20}$. Além dessas conclusões, em relação ao câncer do cólon direito, envolvendo maior incidência relacionada aos aspectos étnicos, sexuais e de faixas etárias, há outros fatores relevantes que devem ser colocados em evidência no curso do diagnóstico dessa neoplasia. São as opiniões que enfatizam as diferenças clínicas e biológicas entre a lesão que cresce nos segmentos esquerdos, e as que se situam no cólon direito, sugerindo mecanismos carcinogenéticos diferentes ${ }^{21,22}$. Relevantes por, eventualmente, poderem ser alvos para cuidados e abordagens terapêuticas diferentes ${ }^{21,23}$.

Outro dado de destaque é que os pacientes com câncer nos segmentos proximais dos cólons, são alvos mais freqüentes para o aparecimento de lesões metacrônicas do que as pessoas que tem o primeiro tumor localizado no cólon esquerdo ${ }^{24}$, fato que, do ponto de vista epidemiológico. pode ser vinculado ao aumento da incidência do câncer do cólon direito e, não só ao melhor prognóstico da lesão localizada nesse segmento anatômico, como também, às possibilidades de diferenças biológicas do comportamento dessas lesões ${ }^{24}$.

Nos termos das considerações sobre o comportamento do câncer de cólon e os sítios anatômicos de crescimento do tumor, o diagnóstico deve ser feito como conseqüência de programas populacionais de rastreamento, viabilizados por projetos bem delineados e acessíveis ${ }^{25}$, não cabendo, portanto o destaque para os sintomas e sinais da doença. Sob aspecto de busca por meio dos sinais e sintomas, por exemplo, dar-se-ia realce para a anemia de etiologia desconhecia, podendo ter como origem o ceco ou cólon ascendente, motivado pelo adenocarcinoma, às vezes, expressado, inclusive, pela massa que o paciente, de repente, sente ao passar a mão no abdômen, durante o banho. Assim é ainda, não raramente, a maneira de diagnosticar tardiamente o câncer de ceco. Contudo, esses sinais e as alterações intestinais funcionais - alteração da consistência das fezes, fragmentação e até a diarréia - dores abdominais, emagrecimento são expressões de doença já avançada.

Os destaques feitos por Goligher ${ }^{10}$ a respeito das manifestações clínicas do câncer dos cólons, so- bretudo quando localizado no ceco ou no cólon ascendente, dão ênfase a quase completa ausência de sinais e sintomas que, quando ocorrem, são representados muito mais por diarréia do que por constipação. Muitas vezes a única manifestação é de um vago comprometimento do estado geral de saúde, com emagrecimento e anemia.

Os sintomas são incertos e intermitentes de tal modo que são facilmente confundidos com os fenômenos dispépticos dolorosos ou não, cujo fator etiológico pode ter relações gastroduodenais, intestinais e colocistopáticas.

Plenitude epigástrica, empachamento, distensão abdominal, eructação, desconforto referidos nos quadrantes direitos, inferior e superior, do abdômen são sintomas que podem estar relacionados às alterações gastrintestinais funcionais, mas, também ao câncer do cólon direito ${ }^{10}$.

\section{ASPECTOS PREVENTIVOS}

As manifestações clínicas insidiosas e as dificuldades inerentes ao mais precoce diagnóstico fundamentam as preocupações que há no sentido de levar em frente às campanhas populares de prevenção do câncer colorretal, cujo teor pode incluir o esclarecimento a respeito dos fatores de riscos - genéticos, alimentares e raciais, entre outros - em todos os programas possíveis que visam prevenir e rastrear o câncer dos cólons.

Assim, a prevenção deve ser será enfocada em primária e secundária e que consistem em: a primária - identificação e eliminação dos agentes carcinogenéticos ambientais - e a secundária no rastreamento de pacientes com mais alto risco de câncer e erradicação das lesões pré-cancerosas ${ }^{7}$, ambas envolvendo a pessoa, de um modo geral, e, em alguns aspectos, englobando estudos populacionais.

\section{a. Prevenção primária}

Estudos da interferência de certas substâncias na modificação do crescimento de lesões pré-cancerosas devem ser considerados quando se pretende formular uma estratégia de prevenção primária.

Há vários elementos, orgânicos e inorgânicos, que entram na composição da alimentação e que recebem destaques por eventuais efeitos no crescimento de lesões neoplásicas e, portanto, são apontados como protetores, mas nem sempre o são, de fato. Por exem- 
plo, o â-caroteno (pró-vitamina A), encontrado em frutos, raízes, tubérculos e legumes, foi indicado pela sua aparente capacidade de diminuir o risco de câncer de pulmão entre os fumantes, todavia em estudos dirigidos o efeito foi contrário.?

No tocante ao câncer de cólon, os estudos de aplicação sobre efeitos de substâncias químicas na remissão da doença têm sido dirigidos para a polipose heredo-familiar (PAF) onde o que se pretendeu estudar foi a remissão dos adenomas - sua diminuição em número ou no tamanho - ou, as vezes, a diminuição do risco de câncer ${ }^{26}$. Os dados obtidos são sugestivos de que as vitaminas antioxidantes (â-caroteno, vitamina $\mathrm{C}$ e vitamina E) não têm qualquer efeito sobre os adenomas e nem na proteção contra o câncer. ${ }^{26}$ Por outro lado, o cálcio tem ação moderada (25\%) sobre o risco de recidiva de adenomas, mas não altera o aumento do tamanho do pólipo. O farelo de trigo não diminui o risco de rediviva de adenomas enquanto as mucilagens, contrário de tudo que se esperava, aumentam.

Nessas condições, o que se tem como consenso é orientar o aumento do consumo de verduras e legumes, diminuir o aporte calórico e aumentar as atividades físicas, medidas, no entanto que não foram avaliadas em estudos apropriados. ${ }^{27}$

Para cada segmento do trata digestório, há uma proposição relativa aos componentes da dieta - de um modo geral o que se preconiza é a diminuição do total de energia consumida, evitando-se gorduras e gorduras saturada, grelhados e defumados com o concomitante aumento da ingestão de frutas, vegetais e fibras, o que pode estar de acordo com alguns estudos sobre o câncer do esôfago. $\mathrm{O}$ adicional consumo de frutas e vegetais incluindo os que têm alto teor de acido ascórbico, pode estar associado a um risco diminuído para o aparecimento do câncer gástrico, mas não há evidências que essas dietas modifiquem o estado da infecção pelo Helicobacter pylori. Por outro lado, não há nenhuma evidência que suporte o papel de dieta rica em fibras ou pobre em gordura na redução dos adenomas dos cólons. ${ }^{28}$

Se os alimentos, de um modo geral, pouco interferem com a carcinogênese cólica, a aspirina e outros antiinflamatórios não esteroidais podem ter ações interessantes, pelos seus efeitos inibitórios sobre as prostaglandinas ${ }^{29}$ com a confirmação de que essas substâncias estariam associadas à diminuição do risco de crescimento de adenomas e de câncer do cólon. ${ }^{30-34}$ Essas citações têm conotação apenas históricas, pois, na realidade, a aspirina não tem qualquer efeito protetor contra o câncer do intestino grosso ${ }^{35,36}$.

No artigo anterior ${ }^{5}$, entre outras substâncias eventualmente protetoras contra o câncer colorretal, foram objetos de destaque a vitamina $\mathrm{D}$, vitamina $\mathrm{C}$, e agressores, como citado acima, as dietas altamente calóricas, tendo como substratos os carboidratos e as gorduras com seus aspectos interessantes e inerentes controvérsias.

A história da dieta gordurosa tem paralelo com a dieta altamente calórica cujos substratos são os carboidratos, bem como com o grau de atividade física e os gastos energéticos. Os alimentos de alto teor energético (açucares e gorduras) poderiam ser relacionados à etiologia do câncer em função de seus requerimentos metabólicos, e as disfunções conseqüentes envolvendo principalmente a resistência à insulina e a intolerância à glicose ${ }^{37,38}$.

No que se refere à alimentação, o que poderia ser conjecturado, exceto para as doenças que de fato tem origem em substâncias contidas nos alimentos (ex. a intolerância ao glúten) ou que se agravam com elas (ex. o diabete) ou outras doenças metabólicas, é muito pouco provável que, numa alimentação equilibrada possa ser destacado algum fator que contribua no sentido de diminuir, exercendo ação protetora; ou aumentar, exercendo ação agressora, a possibilidade da pessoa adquirir um determinado tipo de doença, principalmente o câncer.

As doenças adquiridas por causa do consumo de alimentos são quase todas relacionadas a uma grande variedade de microorganismos patogênicos (vírus, bactérias, fungos, parasitas, etc.), muito mais do que a presença de um elemento, parte constituinte desses alimentos, com propriedades "tóxicas" ou, eventualmente, "protetoras".

$\mathrm{O}$ homem, de seus ancestrais mais primitivo (se são os hominídeos), vem evoluindo há mais de 1 milhão de anos - data do paleolítico primitivo - e, em todos os aspectos, com um infindável número de melhorias, inclusive relacionadas aos alimentos, de tal forma que, embora seja possível, é bem pouco provável que os alimentos que fazem parte do cardápio humano possam estar envolvidos na gênese das doenças neoplásicas.

\section{b. Prevenção secundária}

A identificação das pessoas portadoras de fatores reconhecidamente envolvidos com a gênese do 
câncer, e o conhecimento da genética das doenças que predispõem ao câncer de cólon tais como a polipose heredo-familiar (PAF) e o câncer hereditário não relacionado à polipose (HNPCC) são elementos fundamentais na prevenção secundária.

Esses tumores têm seu desenvolvimento resultante de uma complexa interação entre aspectos genéticos e ambientais. Estima-se, virtualmente, que cerca de 5 a $10 \%$ de todas as neoplasias malignas do intestino grosso têm etiologia primária vinculada à hereditariedade. $\mathrm{O}$ câncer do intestino grosso, não relacionado à polipose hereditária (HNPCC), fica dentro daquela estimativa, variando de 2 a 10\%. O HNPCC é uma doença autossômica que se caracteriza pelo desenvolvimento do câncer do intestino grosso e outros cânceres (endométrio, estômago, intestino delgado, retroperitônio, trato urinário, ovários e cérebro etc. ${ }^{5,36}$ ). Trata-se de um fator de risco, por enquanto inevitável, onde a prevenção se torna possível na medida da facilidade que possa haver para a identificação do fator genético. Por outro lado, na PAF, se a mutação não puder ser identificada, a doença pode ser descoberta pelo exame endoscópico, que deve ser iniciado na puberdade precoce ${ }^{7}$. Nos casos de HNPCC, seja para os portadores do gene e nos que a mutação não foi identificada, a recomendação é fazer o exame coloscópico a cada 2 anos, a partir dos 25 anos ou 5 anos antes da idade mais precoce em que houve o aparecimento do câncer na família ${ }^{37-42}$, incluindo, para as mulheres, o exame ginecológico sistemático com a citologia (aspirativa ou esfregaço) e ultra-som endovaginal, depois dos 25 anos de idade, apesar de algumas controversas ${ }^{43,44}$.

A pessoa que tem dois ou mais parentes de primeiro grau ou parente com câncer antes dos 60 anos de idade deve ser examinado, a cada 5 anos por meio de coloscopia, inicialmente aos 45 anos ou na idade menos 5 anos do parente mais jovem com câncer $39,42,45,46$.

\section{Estudos populacionais (rastreamento)}

O câncer colorretal preenche os critérios de doença que justifica a organização de rastreamento populacional preventivo ${ }^{47-50}$, principalmente aplicados depois das "campanhas populares" por meio de emissoras de rádio, televisão, jornais de grande penetração e distribuição de impressos. Em geral, o rastreamento mais acessível, nessa circunstância, é feito pela pesquisa de sangue oculto nas fezes por meio de teste de sangue oculto nas fezes (Hemoccult ${ }^{\circledR}$ - Beckman Coulter, Inc.) que é de fácil distribuição, manuseio, recuperação e análise de resultados, e tem efeito preventivo efetivo ${ }^{51}$. Contudo, como em todos os programas de incentivo à saúde pública, a aderência é quase sempre decepcionante, mesmo em populações especialmente escolhidas ${ }^{52}$. As pessoas constroem barreiras, às vezes irredutíveis, avaliam benefícios e criam rejeições antes de mudar qualquer tipo de comportamento.

$\mathrm{O}$ reconhecimento desse fato ensejou estudo do perfil da população negra da Carolina do Norte (USA), quanto à adesão, segundo as rejeições e benefícios elencados, diante de procedimentos de campanha de saúde para prevenção do câncer. O projeto foi, também, baseado na observação de que a taxa de mortalidade pelo câncer colorretal (1991-1995) era de 27 e 20,5 por 100.000 , para homens e mulheres afroamericanos, comparado com 20 e 13,5 por 100.000 para homens e mulheres brancos. A suposição inicial foi que os diferentes valores deveriam estar relacionados à menor adesão às campanhas de prevenção primária e secundária e que os números poderiam ser mudados conhecendo-se o caráter comportamental da população alvo. ${ }^{52}$

O projeto chamou WATHC (Wellness for African-Americans Through Churches) com o seguinte significado: "saúde para os afro-americanos através da Igreja". Os boletins informativos e questionários metodicamente elaborados foram distribuídos aos membros de doze Igrejas rurais, durante os cultos semanais, abrangendo uma população de 830 pessoas com mais de 18 anos de idade que depois de vários escrutínios e análises, ficou estabilizada em 397 pessoas com idades iguais ou superiores a 50 anos; $37 \%$ tinham ensino primário, $30 \%$ tinham ensino médio o curso técnico completo e $18 \%$ tinham curso superior.

O projeto foi delineado para motivar, na população eleita, aos seguintes objetivos comportamentais: aumento do consumo de vegetais e frutas, reduzir a ingestão de gorduras, aumento regular das atividades físicas e aumento da participação nos exames de rastreamento do câncer.

Nesse projeto, os autores tentaram, em primeiro, descrever a proporção da amostra populacional estudada que tinha se submetido a exames preventivos para o câncer colorretal e qual a aceitação das normas estabelecidas pelo rastreamento e, em segundo, tentaram investigar a relação entre a percepção de vanta- 
gens e de rejeições ao rastreamento do câncer colorretal.

Os procedimentos preventivos secundários incluídos foram: pesquisa de sangue oculto nas fezes, retossigmoidoscopia flexível e coloscopia ou qualquer associação deles.

As rejeições e benefícios mais comumente percebidos em relação ao projeto de rastreamento de câncer colorretal foram ${ }^{52}$ :

- Rejeições percebidas

1. o médico nunca recomendou o teste $(55 \%)$

2. o teste seria doloroso $(45 \%)$

3. o teste seria muito caro $(12 \%)$

4. o teste seria muito embaraçoso $(22 \%)$

5. preparo para o teste é muito difícil $(20 \%)$

- Vantagens percebidas

$(86 \%)$

1. servirá como bom exemplo para a família

2. para cuidar do corpo que é o templo sagrado de Deus $(85 \%)$

3. para seguir o conselho do médico $(76 \%)$

4. para ter melhor controle sobre a saúde $(75 \%)$

5. para ter menos preocupação $(73 \%)$

Esse tipo de experiência e os resultados auferidos põem em evidência o significado das dificuldades encontradas pelo gestor de saúde, quando se pretende mobilizar a opinião pública e consolidar mu- danças de comportamento. Esses aspectos, por fatores diversos, são mais notáveis nas camadas socioeconômicas de menos recursos. ${ }^{53}$. Na região norte da Europa a adesão tem sido superior a 55\%, imediatamente após as campanhas - 55\% na Inglaterra, $66 \%$ na Suécia e $57 \%$ na Dinamarca), mas não é o que ocorre na França, onde a taxa de participação não passava de $20 \%$ 54, 55 .

O meio inicial de rastreamento, feito pela pesquisa de sangue oculto nas fezes endereçado para a população acima de 50 anos de idade, com os reconhecidos benefícios ${ }^{56-58}$, é o mais acessível e de mais alta especificidade, apesar da sensibilidade variável e de estar sendo substituído pelo exame endoscópico seja a retossigmoidoscopia flexível ou a coloscopia. $\mathrm{O}$ teste permite detectar cerca de $50 \%$ dos cânceres e $20 \%$ dos adenomas com mais de um centímetro. Com isso, a redução da mortalidade esperada varia de 15 a $18 \%$, em 7 a 10 anos, destacando a importância da aplicação sistemática do teste no rastreamento populacional do câncer colorretal ${ }^{56-59}$.

Em suma, o câncer dos cólons, altamente prevalente, pode ser tratado e curado quando precocemente diagnosticado. Nessas circunstâncias, a estimativa de cura acima de $90 \%$, feita há 60 anos, não foi significativamente modificada com as aderências de novos e de mais modernos procedimentos terapêuticos. Isso nos endereça ao maior esforço conjunto no sentido de estimular, cada vez mais, o rastreamento preventivo populacional do câncer colorretal.

ABSTRACT: The colorectal cancer is the third most common cancer diagnosed and is the second leading cause of cancer death among American people. However, when it is detected in early stages, it has high level of cure with 5-year survival over 95\%. So, colorectal cancer must be precociously diagnosed and surgically managed. Preventive strategy must be done using fecal occult blood test for mass colorectal screening.

Key words: cancer screening, preventive strategy, fecal occult blood.

\section{REFERÊNCIAS}

1. Society AC. Cancer Facts \& Figures. 2008. pp. 12-13.

2. Cress RD, Morris C, Ellison GL, Goodman MT. Secular changes in colorectal cancer incidence by subsite, stage at diagnosis, and race/ethnicity, 1992-2001. Cancer 2006; 107(5 Suppl):1142-52.

3. Ferlay J, Autier P, Boniol M, et al. Estimates of the cancer incidence and mortality in Europe in 2006. Ann Oncol 2007; 18(3):581-92.
4. Cancer MdSI-INd. Incidência de Cancer no Brasil - Estimativa de 2008. Vol. 2008. Rio de janeiro, 2008.

5. Santos Jr JCM. Câncer ano-reto-cólico: Aspectos atuais II Câncer colorretal: fatores de riscos e prevenção. Rev bras Coloproctol 2007; 27(4):459-473.

6. Society AC. Cancer Facts \& Figures. 2007. pp. 1-56.

7. Fabre E, Spano JP, Atlan D, et al. [Cancer of the colon: an update]. Bull Cancer 2000; Suppl 4:5-20.

8. Abrapeci. Associação Brasileira de Prevenção do Câncer do Intestino. 2008. 
9. Vainio H, Miller AB. Primary and secondary prevention in colorectal cancer. Acta Oncol 2003; 42(8):809-15.

10. Goligher J. Surgery of the Anus, Rectum and Colon. In John Goligher, Hebert Duthie, Nixon H, eds. London: Baillière Tindall, 1984. pp. 1161.

11. Gomez D, Dalal Z, Raw E, et al. Anatomical distribution of colorectal cancer over a 10 year period in a district general hospital: is there a true "rightward shift"? Postgrad Med J 2004; 80(949):667-9.

12. Patel K, Hoffman NE. The anatomical distribution of colorectal polyps at colonoscopy. J Clin Gastroenterol 2001; 33(3):222-5.

13. Obrand DI, Gordon PH. Continued change in the distribution of colorectal carcinoma. Br J Surg 1998; 85(2):246-8.

14. Erkek B, Ozkan N, Bayar S, et al. Subsite distribution of colorectal carcinoma and implications for screening; a retrospective audit of 1771 cases. Hepatogastroenterology 2007; 54(73):77-80.

15. Takada H, Ohsawa $\mathrm{T}$, Iwamoto $\mathrm{S}$, et al. Changing site distribution of colorectal cancer in Japan. Dis Colon Rectum 2002; 45(9):1249-54.

16. McFarlane ME, Rhoden A, Fletcher PR, Carpenter R. Cancer of the colon and rectum in a Jamaican population: diagnostic implications of the changing frequency and subsite distribution. West Indian Med J 2004; 53(3):170-3.

17. Mensink PB, Kolkman JJ, Van Baarlen J, Kleibeuker JH. Change in anatomic distribution and incidence of colorectal carcinoma over a period of 15 years: clinical considerations. Dis Colon Rectum 2002; 45(10):1393-6.

18. Nelson RL, Dollear T, Freels S, Persky V. The relation of age, race, and gender to the subsite location of colorectal carcinoma. Cancer 1997; 80(2):193-7.

19. Qing SH, Rao KY, Jiang HY, Wexner SD. Racial differences in the anatomical distribution of colorectal cancer: a study of differences between American and Chinese patients. World J Gastroenterol 2003; 9(4):721-5.

20. Shavers VL. Racial/ethnic variation in the anatomic subsite location of in situ and invasive cancers of the colon. J Natl Med Assoc 2007; 99(7):733-48.

21. Kurome M, Kato J, Nawa T, et al. Risk factors for high-grade dysplasia or carcinoma in colorectal adenoma cases treated with endoscopic polypectomy. Eur J Gastroenterol Hepatol 2008; 20(2):111-7.

22. Nawa T, Kato J, Kawamoto H, et al. Differences between right- and left-sided colon cancer in patient characteristics, cancer morphology and histology. J Gastroenterol Hepatol 2008; 23(3):418-23.

23. Sarli L, Michiara M, Sgargi P, et al. The changing distribution and survival of colorectal carcinoma: an epidemiological study in an area of northern Italy. Eur J Gastroenterol Hepatol 2005; 17(5):567-72.

24. Gervaz P, Bucher P, Neyroud-Caspar I, et al. Proximal location of colon cancer is a risk factor for development of metachronous colorectal cancer: a population-based study. Dis Colon Rectum 2005; 48(2):227-32.

25. Almeida FF, Araujo SE, Santos FP, et al. Colorectal cancer screening. Rev Hosp Clin Fac Med Sao Paulo 2000; 55(1):3542.

26. Faivre J, Bonithon-Kopp C. Chemoprevention of colorectal cancer. Recent Results Cancer Res 1999; 151:122-33.

27. Conférence de consensus. Prévention, dépistage et prise en charge des cancers du côlon. Gastroenterol Clin Biol 1999; 22:5275-88.

28. Thomson CA, LeWinn K, Newton TR, et al. Nutrition and diet in the development of gastrointestinal cancer. Curr Oncol Rep 2003; 5(3):192-202.

29. Kargman SL, O'Neill GP, Vickers PJ, et al. Expression of prostaglandin G/H synthase-1 and -2 protein in human colon cancer. Cancer Res 1995; 55(12):2556-9.

30. Benamouzig R. [Do aspirin or nonsteroidal anti-inflammatory drugs decrease the risk of colorectal cancer?]. Gastroenterol Clin Biol 1998; 22(3 Suppl):S22-7.

31. Kune GA, Kune S, Watson LF. Colorectal cancer risk, chronic illnesses, operations, and medications: case control results from the Melbourne Colorectal Cancer Study. Cancer Res 1988; 48(15):4399-404.

32. Rosenberg L, Palmer JR, Zauber AG, et al. A hypothesis: nonsteroidal anti-inflammatory drugs reduce the incidence of large-bowel cancer. J Natl Cancer Inst 1991; 83(5):355-8.

33. Peleg, II, Maibach HT, Brown SH, Wilcox CM. Aspirin and nonsteroidal anti-inflammatory drug use and the risk of subsequent colorectal cancer. Arch Intern Med 1994; 154(4):394-9.

34. Muscat JE, Stellman SD, Wynder EL. Nonsteroidal antiinflammatory drugs and colorectal cancer. Cancer 1994; 74(7):1847-54.

35. Gann PH, Manson JE, Glynn RJ, et al. Low-dose aspirin and incidence of colorectal tumors in a randomized trial. J Natl Cancer Inst 1993; 85(15):1220-4.

36. Giovannucci E, Egan KM, Hunter DJ, et al. Aspirin and the risk of colorectal cancer in women. N Engl J Med 1995; 333(10):609-14.

37. Vasen HF, Mecklin JP, Khan PM, Lynch HT. The International Collaborative Group on HNPCC. Anticancer Res 1994; 14(4B):1661-64.

38. Vasen HF, Watson P, Mecklin JP, Lynch CF. New clinical criteria for hereditary nonpolyposis colorectal cancer (HNPCC, Lynch syndrome) proposed by the International Collaborative group on HNPCC. Gastroenterology 1999; 116(6):1453-56.

39. Dunkop M. Guidance on gastrointestinal surveillance for hereditary non-polyposis colorectal cancer, familial adenomotous polyposis, juvenile polyposis, and PeutzJeghers syndrome. Gut 2002; 51(5):12-27.

40. Halbert CH, Lynch H, Main D, et al. Colon Cancer Screening Practices Following Genetic Testing for Hereditary 
Nonpolyposis Colon Cancer (HNPCC) Mutations Arch Intern Med 2004; 164(17):1881-87.

41. Umar A, Boland CR, Terdiman JP, et al. Revised Bethesda Guidelines for hereditary nonpolyposis colorectal cancer (Lynch syndrome) and microsatellite instability. J Natl Cancer Inst 2004; 96(4):261-68.

42. Dove-Edwin I, Sasieni P, Adams J, Thomas HJW. Prevention of colorectal cancer by colonoscopic surveillance in individuals with a family history of colorectal cancer: 16 year, prospective, follow-up study. BMJ 2005; 331:1047-49.

43. Brown GJE, Aittomäki K. Cancer Risk in Young Women at Risk of Hereditary Nonpolyposis Colorectal Cancer: Implications for Gynecologic Surveillance. Gynecol Oncology 2001; 80(3):346-49.

44. Dove-Edwin I, Boks D, Goff S, al. e. The outcome of endometrial carcinoma surveillance by ultrasound scan in women at risk of hereditary nonpolyposis colorectal carcinoma and familial colorectal carcinoma. Cancer 2002; 94(6):170812.

45. Winawer SJ, O'Brien MJ, Waye JD, et al. Risk and surveillance of individuals with colorectal polyps. Bull WHO 1990; 68:7789.

46. de Jong AE, Morreau H, Nagengast FM, et al. Prevalence of Adenomas Among Young Individuals at Average Risk for Colorectal Cancer. Am J Gastroenterol. 2005; 100(1):139-43.

47. Miller MP, Stanley TV. Results of a mass screening program for colorectal cancer. Arch Surg 1988; 123(1):63-65.

48. Towler BP, Irwig L, Glasziou P, et al. Screening for colorectal cancer using the faecal occult blood test, hemoccult. Cochrane Database Syst Rev 2000; 1(CD001216).

49. Faivre J. State of the art in colorectal cancer screening. Gut 2000; 47(Suppl 3):A38.

50. Heresbach D, Manfredi S, D'halluin PN, et al. Review in depth and meta-analysis of controlled trials on colorectal cancer screening by faecal occult blood test. Eur J Gastroenterol Hepatol 2006; 18(4):427-33.

51. Mandel JS, Church TR, Bond JH, et al. The effect of fecal occult blood screening on the incidence of colorectal cancer. $\mathrm{N}$ Engl J Med 2000; 343(22):1603-7.
52. James AS, Campbell MK, Hudson MA. Perceived barriers and benefits to colon cancer screening among african americans in North Carolina: How does perception relate to screening behavior? Cancer Epidemiol, Biomarkers \& Prevention 2002; 11:529-34.

53. Nagamine M, Phillips K, Haas J, Liang SY. Predictors of Utilization of Colorectal Cancer Screening Tests (CRC) and Adherence to the Screening Guidelines among Low-Income Populations. Abstr AcademyHealth Meet 2005; 22(abst 4007):4007.

54. Launoy G, Herbert C, Vallee JP, et al. [Mass screening for colorectal cancer in France. Experience in 165,000 people in the department of Calvados]. Gastroenterol Clin Biol 1996; 20(3):228-36.

55. Flachs A. [Self-evaluation of the dynamics of distribution of the Hemoccult II test in the practice of general medicine for departmental mass screening]. Gastroenterol Clin Biol 1995; 19(4):457-8.

56. Bretagne JF, Manfredi S, Heresbach D. Colorectal cancer mass screening: present and future. Presse Med 2007; 37:363-66.

57. Lindholm E, Brevinge $\mathrm{H}$, Haglind E. Survival benefit in a randomized clinical trial of faecal occult blood screening for colorectal cancer. Br J Surg 2008; 95(8):1029-36.

58. Hewitson P, Glasziou P, Watson E, et al. Cochrane systematic review of colorectal cancer screening using the fecal occult blood test (hemoccult): an update. Am J Gastroenterol 2008; 103(6):1541-49.

59. Faivre J, Tazi MA, Milan C, et al. Controlled trial of faecal occult blood screnning for colorectal cancer in Burgundy (Franc): results of the first 9 years. Gastroenterology 1999; 116:A400.

\section{Endereço para correspondência:} JÚLIO CÉSAR M SANTOS JR Instituto de Medicina

Av. Ministro Urbano Marcondes, 516

12515-230 Guaratinguetá, SP

E-mail: instmed@provale.com.br 